\title{
Downregulation of LPXN expression by siRNA decreases the malignant proliferation and transmembrane invasion of SHI-1 cells
}

\author{
GUO-HUA ZHU ${ }^{1}$, HAI-PING DAI ${ }^{2}$, QUN SHEN ${ }^{1,3}$ and QI ZHANG ${ }^{1}$ \\ ${ }^{1}$ First Clinical College, Nanjing University of Chinese Medicine, Nanjing, Jiangsu 210023; \\ ${ }^{2}$ Leukemia Research Unit, Jiangsu Institute of Hematology, First Affiliated Hospital of Soochow University, Suzhou, \\ Jiangsu 215006; ${ }^{3}$ Department of Hematology, First Affiliated Hospital of Nanjing University of Chinese Medicine, \\ Nanjing, Jiangsu 210009, P.R. China
}

Received December 27, 2017; Accepted August 30, 2018

DOI: $10.3892 / \mathrm{ol} .2018 .9605$

\begin{abstract}
The aim of the present study was to investigate the effects of decreasing leupaxin (LPXN) expression on the proliferation and invasion of human acute monocytic leukemia SHI-1 cells. The transfection efficiency of fluorescein amidite (FAM)-small interfering RNA (siRNA) was determined using flow cytometry, and the protein expression levels of LPXN, phosphorylated (p)-c-Jun N-terminal kinase (JNK), p-p38 mitogen-activated protein kinase (p38 MAPK) and p-extracellular-signal-regulated kinase (ERK) were detected by western blot analysis. Proliferation was determined using the cell counting kit- 8 reagent and cellular transmembrane invasion ability was determined using a Transwell chamber system. The gelatinase levels of matrix metalloproteinase (MMP)-2 and MMP-9 in the cell culture supernatant were also analyzed by gelatin zymography. In SHI-1 cells, the optimal transfection conditions of siRNA were a cell density of $4 \times 10^{5}$ cells $/ \mathrm{ml}$ and a ratio of siRNA/Lipofectamine ${ }^{\circledR} 2000$ of $200 \mathrm{pmol} / 1 \mu \mathrm{l}$. The highest transfection efficiency of FAM-siRNA was $74.5 \%$. In the present study, L2-siRNA was selected to effectively decrease the expression of LPXN. Following downregulation of LPXN expression by L2-siRNA, proliferation inhibition rates increased to $27.043 \pm 2.051$ and cell transmembrane invasion rates decreased to $25.270 \pm 2.145(\mathrm{P}<0.05)$. The results of the western blot analysis and the gelatin zymography indicated that downregulation of LPXN expression increased the expression of p-p38 MAPK and p-JNK, and attenuated the secretion levels of MMP-2 and MMP-9. However, downregulation of LPXN expression had no effect on p-ERK expression in SHI-1 cells. The results of the present study indicated that
\end{abstract}

Correspondence to: Dr Guo-Hua Zhu, First Clinical College, Nanjing University of Chinese Medicine, 138 Xianlin Road, Nanjing, Jiangsu 210023, P.R. China

E-mail: gloria0601@njucm.edu.cn

Key words: leupaxin, small interfering RNA, SHI-1 cell downregulation of LPXN expression decreased the malignant proliferation and transmembrane invasion of SHI-1 cells by activating JNK and p38 MAPK, and inhibiting MMP-2 and MMP-9 secretion.

\section{Introduction}

Leupaxin (LPXN) was cloned from a human macrophage cDNA library and was identified as a new member of the paxillin family by Lipsky et al in 1998 (1). The relative molecular mass of LPXN is $43 \mathrm{kDa}$, and the protein is primarily expressed in the cellular cytoplasm of leukemia, prostate cancer, breast cancer, melanoma and other types of tumor cells (2). Similar to paxillin, LPXN is localized in the focal adhesion plaque (FAP), serving as a molecular adaptor involved in integrin-mediated signaling and as a regulator for proliferation, differentiation, adhesion and migration (3).

A number of systematic and in-depth studies on LPXN and prostate cancer have been performed. In the prostate cancer cell lines PC-3, DU 145 and LNCaP, it has been identified that the expression levels of LPXN were associated with the degree of malignancy of the cells $(4,5)$. Upregulating LPXN expression has been revealed to promote invasion and metastasis of prostate cancer cells, whereas downregulating LPXN expression by RNA interference (RNAi) has been revealed to stimulate the isolation and spontaneous apoptosis of the aforementioned cancer cells (6). Similarly, Chen et al (2) identified that increased expression of LPXN promoted the migration of MDA-MB-231 breast cancer cells to the extracellular matrix.

The association of LPXN and leukemia has also gained increasing attention, as Petti et al (7) utilized the thiophene kinase inhibitor OSI-930 to selectively inhibit tyrosine phosphorylation of LPXN, p130 ${ }^{\text {Cas }}$ and focal adhesion kinase (FAK), which led to apoptosis of the HMC-1 mast cell leukemia line. Tanaka et al (8) revealed that when LPXN was expressed in human leukocytic K562 cells, LPXN significantly suppressed integrin $\alpha 5 \beta 1$-mediated cell adhesion to fibronectin and inhibited tyrosine phosphorylation of paxillin. In addition, Dai et al (9) observed that LPXN was fused to runt-related transcription factor 1 in a patient with 
acute myeloid leukemia with a $\mathrm{t}(11 ; 21)(\mathrm{q} 12 ; \mathrm{q} 22)$ translocation. Additionally, Abe et al (10) reported the generation of the ETV6-LPXN fusion transcript by $\mathrm{t}(11 ; 12)(\mathrm{q} 12.1 ; \mathrm{p} 13)$ in a patient with relapsing acute myeloid leukemia and indicated that ETS variant 6-LPXN serves a crucial function in leukemia progression. Taken together, the aforementioned results indicated that the expression and phosphorylation of LPXN promote proliferation, invasion and metastasis of leukemia cells, and also an association with the occurrence and the development of leukemia.

To the best of our knowledge, the present study is the first attempt to downregulate LPXN expression by RNAi and to investigate the possible downstream effects and molecular mechanisms on proliferation and invasion of the human acute monocytic leukemia SHI-1 cell line in vitro. An increased protein expression level of LPXN in SHI-1 cells has been reported, in addition to higher secretion levels of matrix metalloproteinase (MMP)-2 and MMP-9 gelatinase, where a marked migration ability and tumorigenicity has been observed in nude mice (11).

\section{Materials and methods}

Reagents. Iscove's modified Dulbecco's medium (IMDM), Opti-MEM $^{\circledR}$ I reduced serum medium and fibronectin were purchased from Gibco (Thermo Fisher Scientific, Inc., Waltham, MA, USA). Cell Counting Kit-8 (CCK-8) was purchased from Dojindo Molecular Technologies, Inc. (Kumamoto, Japan). Matrigel and Transwell chambers were purchased from BD Biosciences (Franklin Lakes, NJ, USA). The antibodies used were against LPXN (dilution, 1:500; ab67571; Abcam, Cambridge, UK), $\beta$-actin (dilution, 1:3,000; \#4967S; Cell Signaling Technology, Inc. Danvers, MA, USA) and p-mitogen-activated protein kinase (p-MAPK; dilution, 1:1,000; \#9910; Cell Signaling Technology, Inc.). The small interfering RNA (siRNA) sequences of fluorescein amidite (FAM)-siRNA, LPXN-siRNA and luciferase-siRNA were synthesized by Shanghai GenePharma Co., Ltd. (Shanghai, China).

Cell culture. The leukemia cell line SHI-1 was gifted by the Jiangsu Institute of Haematology, First Affiliated Hospital of Soochow University (Suzhou, China) and was originally derived from the mononuclear cells of the bone marrow from a patient with acute monocytic leukemia in relapse. The cells were maintained as a stable cell line in vitro (11). SHI-1 cells were incubated in IMDM with $15 \%$ fetal bovine serum (Gibco; Thermo Fisher Scientific, Inc.) at $37^{\circ} \mathrm{C}$ with $5 \% \mathrm{CO}_{2}$. Cells in the exponential growth phase following passaging every 3-4 days were used for the subsequent experiments.

Transfection of SHI-1 cells with siRNA. Specific siRNAs of the LPXN gene were designed according to a previously described protocol (12). Transfection of SHI-1 cells was accomplished using Lipofectamine ${ }^{\circledR} 2000$ (Invitrogen; Thermo Fisher Scientific, Inc.) according to the manufacturer's protocol with the following different LPXN gene-specific siRNA duplexes: L1-siRNA, sense, 5'-UAUUCCAACCCAGCUCCUC-3' and antisense, 5'-GAGGAGCUGGGUUGGAAUA-3'; L2-siRNA, sense, 5'-GGCGCAGCUCGUGUAUACUACCAAU-3' and antisense, 5'-AUUGGUAGUAUACACGAGCUGCGCC-3' (Shanghai GenePharma Co., Ltd.). The negative control group was transfected with siRNA duplex oligonucleotides against the firefly luciferase gene (N-siRNA) (Shanghai GenePharma Co., Ltd.) and the positive control group was transfected with FAM-siRNA in SHI-1 cells.

To improve the transfection efficiency, several modifications to the transfection protocol were performed. Briefly,

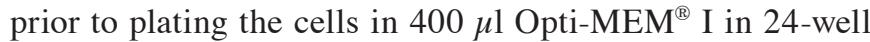
dishes, 4x10 ${ }^{5}$ SHI-1 cells were washed with serum-free IMDM. Prior to combining the reagents, the siRNA oligomer and Lipofectamine 2000 were diluted in $50 \mu \mathrm{l}$ Opti-MEM ${ }^{\circledR} \mathrm{I}$. The transfection efficiency was assessed with FAM-siRNA using flow cytometry (FCM), as previously described by Wang et al (11).

Western blot analysis. According to the manufacturer's protocol of Membrane and Cytosol Protein Extraction kit (Beyotime Institute of Biotechnology, Nanjing, China), total cellular extracts were collected and the protein concentration was determined after transfection $48 \mathrm{~h}$. Equal amounts of extracted proteins were subjected to western blotting and the specific experiments were conducted as previously described (13). Following SDS-PAGE (5\% concentration gel and $10 \%$ separation gel) electrophoresis and membrane transfer to polyvinylidene fluoride membranes (EMD Millipore, Billerica, MA, USA), the proteins were blocked with 5\% bovine serum albumin (Beyotime Institute of Biotechnology) at room temperature for $2 \mathrm{~h}$ and then incubated with the primary antibodies against LPXN (Abcam), phosphorylated (p)-c-Jun, N-terminal kinase (p-JNK), p-p38 MAPK, p-extracellular-signal-regulated kinase (p-ERK) (Phospho-MAPK Family Antibody Sampler kit; dilution, 1:1,000; cat. no. 9910) and $\beta$-actin (dilution, 1:3,000; cat. no. 4967S) (both from Cell Signaling Technology, Inc.) at $4^{\circ} \mathrm{C}$ overnight separately. The membranes were washed three times in TBS with $0.1 \%$ Tween-20 and subsequently probed with HRP-conjugated goat anti-mouse IgG (dilution, 1:10,000; cat. no. 7072; Cell Signaling Technology, Inc.) at room temperature for $2 \mathrm{~h}$. Following incubation, the blots were washed three times in TBS with $0.1 \%$ Tween-20, enhanced chemiluminescent reagents (Beyotime Institute of Biotechnology) were added and scanned with an ImageQuant LAS 4000 Mini (GE Healthcare, Chicago, IL, USA).

CCK-8 assay. A CCK-8 assay was used to determine proliferation according to the manufacturer's protocol. SHI-1 cells were transfected with siRNA against LPXN and the luciferase gene, serving as the negative control, as aforementioned. Following incubation with the transfection medium at room temperature. for $24 \mathrm{~h}$, cells were harvested and seeded in a 96-well plate at $4 \times 10^{5}$ cells/well with three wells of each group. Cells were incubated for an additional $48 \mathrm{~h}$ and the spectrophotometric absorbance of each sample was determined at a wavelength of $450 \mathrm{~nm}$, following the addition of $10 \%$ CCK-8 for $3 \mathrm{~h}$.

Cell invasion assay. A Costar Transwell chamber system was used to determine cell invasion. Membrane filters with a pore size of $8 \mu \mathrm{m}$ and a diameter of $6.5 \mathrm{~mm}$ were coated with $50 \mu \mathrm{l}$ Matrigel and subsequently placed into the 

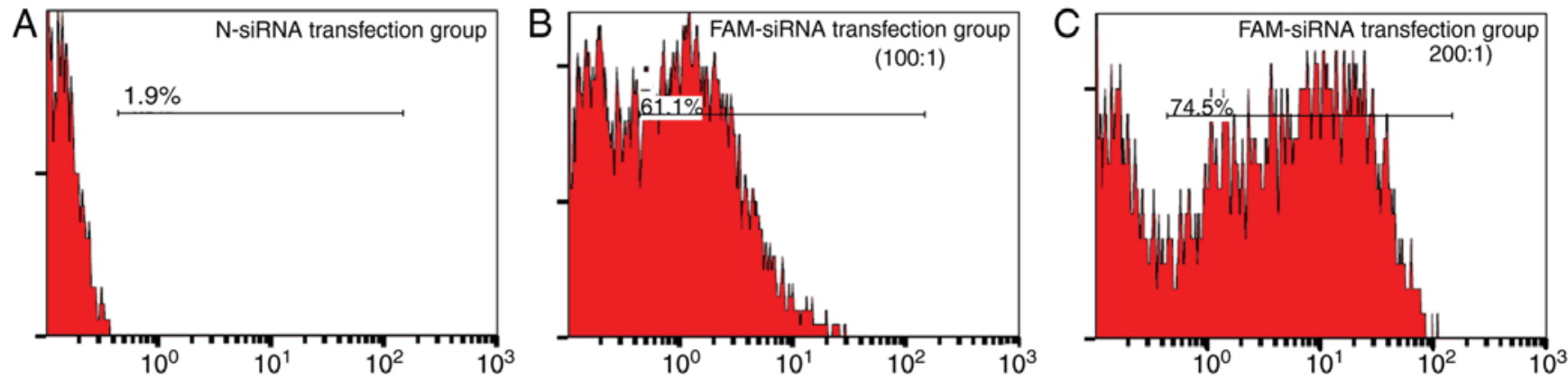

Figure 1. Transfection efficiency of FAM-siRNA into SHI-1 cells detected by flow cytometry. (A) Negative control group transfected with siRNA duplex oligonucleotides against the firefly luciferase gene in SHI-1 cells at a cell density of 4x105/ml and a N-siRNA/Lipofectamine $2000 \mathrm{ratio}$ of $200 \mathrm{pmol} / 1 \mu \mathrm{l}$. Transfection efficiency was $1.9 \%$. (B) Positive FAM-siRNA transfection group at a cell density of 4x10\% $/ \mathrm{ml}$ and a FAM-siRNA/Lipofectamine 2000 ratio of $100 \mathrm{pmol} / 1 \mu 1$. Transfection efficiency was $61.1 \%$. (C) Positive FAM-siRNA transfection group at a cell density of $4 \times 10^{5} / \mathrm{ml}$ and a FAM-siRNA/Lipofectamine 2000 ratio of $200 \mathrm{pmol} / 1 \mu \mathrm{l}$. Transfection efficiency was 74.5\%. FAM, fluorescein amidite; siRNA, small interfering RNA; N, negative control.

wells forming the upper chamber. Following transfection for $24 \mathrm{~h}$, the siRNA-treated SHI-1 cells were suspended in $200 \mu \mathrm{l}$ serum-free IMDM and were seeded at a final density of $2.0 \times 10^{5}$ cells/well in the upper chamber. The lower chamber of invasion was filled with $800 \mu 1$ IMDM, supplemented with $10 \%$ human serum, containing $100 \mathrm{ng} / \mathrm{ml}$ stromal-cell-derived factor $1-\alpha$ as a source of chemoattractants. The chambers were incubated for $24 \mathrm{~h}$ at $37^{\circ} \mathrm{C}$ with $5 \% \mathrm{CO}_{2}$. The cells which had migrated into the lower compartment were counted by inverted optical microscope (magnification, x400; Leica Microsystems GmbH, Wetzlar, Germany) and the invasion rate was calculated by comparing the cell number in the lower compartment medium with the total number of leukemia cells, $2.0 \times 10^{5}$ cells, loaded into the upper compartment.

Gelatin zymography. Cell culture supernatants were analyzed for the presence of the secreted MMP-2 and MMP-9 gelatinases using zymography. SHI-1 cells were transfected with siRNA against LPXN and the luciferase gene, serving as the negative control, as aforementioned. Following incubation with the transfection medium for $24 \mathrm{~h}$, SHI-1 cells were harvested and seeded in a 96 -well plate at $4 \times 10^{5}$ cells/well with serum-free IMDM. The supernatant of each sample was harvested after the $24 \mathrm{~h}$ incubation. The samples were separated by SDS-PAGE ( $10 \%$ gels) copolymerized with $0.1 \%$ gelatin (Sigma-Aldrich; Merck KGaA, Darmstadt, Germany) at $4^{\circ} \mathrm{C}$ and were incubated at $37^{\circ} \mathrm{C}$ with developing buffer containing $0.02 \%$ Brij-35 (Sigma-Aldrich; Merck KGaA), according to our previously published protocol (13). Gelatinase-digested regions were visualized as light bands against a blue background at $37^{\circ} \mathrm{C}$ under natural light.

Statistical analyses. All experiments were repeated at least three times and the data were expressed as the mean \pm standard deviation. The image processing software Photoshop CS3 (Adobe Systems Inc., San Jose, CA, USA) and statistical software SPSS (version 15.0; SPSS, Inc., Chicago, IL, USA) was used for analysis. Student's t-test was used to compare the means of two groups and one-way analysis of variance was used to compare means of multiple samples followed by the Least-Significant-Difference and Dunnett's post-hoc test. $\mathrm{P}<0.05$ was considered to indicate a statistically significant difference.

\section{Results}

FAM-siRNA transfection of SHI-1 cells. Among the repeated experiments, the highest transfection efficiency reached $74.5 \%$, when the SHI-1 cells were seeded at a density of $4 \times 10^{5}$ cells $/ \mathrm{ml}$ and the mixing ratio of FAM-siRNA/Lipofectamine 2000 was $200 \mathrm{pmol} / 1 \mu \mathrm{l}$ (Fig. 1). Therefore, these conditions were used for transfection of LPXN siRNA in SHI-1 cells.

L2-siRNA downregulates LPXN expression in SHI-1 cells. After $48 \mathrm{~h}$ of transient transfection of L1-siRNA and L2-siRNA, the protein expression of LPXN was detected using western blot analysis. As indicated in lane $1(200 \mathrm{pmol} / 1 \mu \mathrm{l})$ and lane 2 (100 pmol/1 $\mu \mathrm{l}$ ) in Fig. 2, transfection of L1-siRNA had no effect on the expression of the LPXN protein. Similarly, lane 3 $(200 \mathrm{pmol} / 1 \mu \mathrm{l})$ and lane $4(100 \mathrm{pmol} / 1 \mu \mathrm{l})$ of the N-siRNA, negative control group, did not change LPXN expression levels. In contrast, transfection of L2-siRNA, indicated in lanes $5(200 \mathrm{pmol} / 1 \mu \mathrm{l})$ and $6(100 \mathrm{pmol} / 1 \mu \mathrm{l})$, downregulated LPXN expression in SHI-1 cells compared with the negative control group. In particular, when the transfection ratio of L2-siRNA/Lipofectamine 2000 was $200 \mathrm{pmol} / 1 \mu \mathrm{l}$, LPXN expression was effectively downregulated, as indicated in lane 5. Therefore, in subsequent experiments, L2-siRNA was transfected at the optimal ratio of 200 pmol L2-siRNA/1 $\mu 1$ Lipofectamine 2000 to investigate the effect of LPXN on the proliferation and invasion of SHI-1 cells.

Downregulating LPXN expression decreases SHI-1 proliferation and transmembrane invasion. The effective L2-siRNA and N-siRNA negative control were transfected into SHI-1 cells at a ratio of $200 \mathrm{pmol}$ siRNA/1 $\mu \mathrm{l}$ Lipofectamine 2000. The proliferation of SHI-1 cells was determined using the CCK- 8 assay followed by transient transfection for $48 \mathrm{~h}$. The inhibition proliferation rate of the L2-siRNA transfection group was $27.043 \pm 2.051 \%$, compared with that of the negative control group of $8.247 \pm 1.003 \%$ (Fig. 3). In addition, there was a significant difference between these two groups $(\mathrm{P}<0.05)$, which indicated that transfection with L2-siRNA decreased the proliferation rate in SHI-1 cells by downregulating LPXN expression.

Furthermore, following transfection with L2-siRNA and $\mathrm{N}$-siRNA for $24 \mathrm{~h}$, the SHI-1 cells were collected and plated 


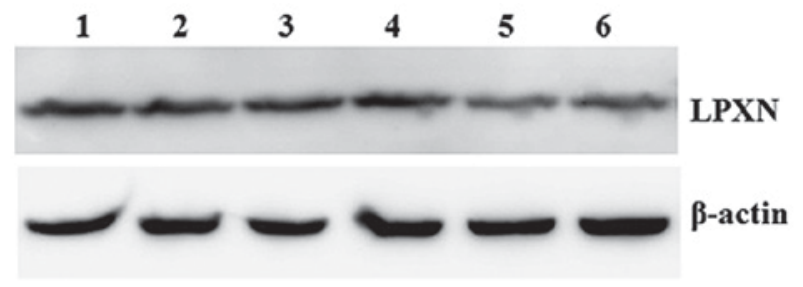

Figure 2. Effects of two different siRNAs, L1-siRNA and L2-siRNA, on LPXN expression in SHI-1 cells detected by western blot analysis. LPXN expression was downregulated when the transfection ratio of L2-siRNA/Lipofectamine 2000 was $200 \mathrm{pmol} / 1 \mu 1$. Lane 1, siRNA/Lipofectamine 2000 at $200 \mathrm{pmol} / 1 \mu \mathrm{l}$; lane 2 , L1-siRNA/Lipofectamine2000at100pmol/1 $\mu$ l;lane3,N-siRNA/Lipofectamine 2000 at $200 \mathrm{pmol} / 1 \mu 1$; lane $4, \mathrm{~N}$-siRNA/Lipofectamine 2000 at $100 \mathrm{pmol} / 1 \mu \mathrm{l}$; lane 5, L2-siRNA/Lipofectamine 2000 at $200 \mathrm{pmol} / 1 \mu \mathrm{l}$; lane 6, L2-siRNA/Lipofectamine 2000 at $100 \mathrm{pmol} / 1 \mu \mathrm{l}$. siRNA, small interfering RNA; LPXN, leupaxin.

in Transwell chambers to determine the transmembrane invasion rate of each group. The transmembrane invasion rate of the L2-siRNA transfection group was $25.270 \pm 2.145$ and the invasion rate of the N-siRNA control group was $36.112 \pm 2.103$ (Fig. 3). The transfection efficiency of siRNA in suspension cells was not high; however, the results of the present study indicated that downregulating the expression of LPXN by L2-siRNA decreased the invasion of SHI-1 cells.

Downregulating the expression of LPXN decreases the gelatinase levels of MMP-2 and MMP-9 in the culture supernatant of SHI-1 cells. To investigate further the effect of LPXN on invasion, the culture supernatant of SHI-1 cells transfected with L2-siRNA and N-siRNA was collected, and the gelatinase level was analyzed by gelatin zymography. There were two white bands in the gelatin, which were identified as MMP-2 (72 kDa) and MMP-9 (92 kDa) (Fig. 4). The gelatinase levels of MMP-2 and MMP-9 between the negative control group in lane 2, transfected with N-siRNA, and the blank control group in lane 1, without any siRNA, remained relatively unchanged (Fig. 4). However, the gelatinase levels in the supernatant of the L2-siRNA transfection group in lane 3 were decreased compared with the aforementioned control groups (Fig. 4). Previous studies have indicated that MMP-2 and MMP-9 serve important functions in the invasion process of various tumors $(11,13)$. Therefore, these results indicated that downregulating LPXN expression by L2-siRNA potentially decreased the secretion of MMP-2 and MMP-9, weakening the invasion of SHI-1 cells.

Downregulating LPXN expression activates p-JNK and p-p38 $M A P K$. MAPKs are serine/threonine protein kinases that have been reported to serve an important function in extrinsic and intrinsic signal transduction pathways, and regulate proliferation, apoptosis and invasion (13). In the present study, the protein expression of p-ERK, p-JNK and p-p38 MAPK were detected by western blotting after $48 \mathrm{~h}$ of transient transfection with L2-siRNA and N-siRNA. In the blots presented in Fig. 5A, lane 1 was the blank control group without any siRNA, lane 2 was the negative control group with N-siRNA transfection and lane 3 was the L2-siRNA transfection group. The expression level of $\mathrm{p}-\mathrm{ERK}$ in these three groups remained

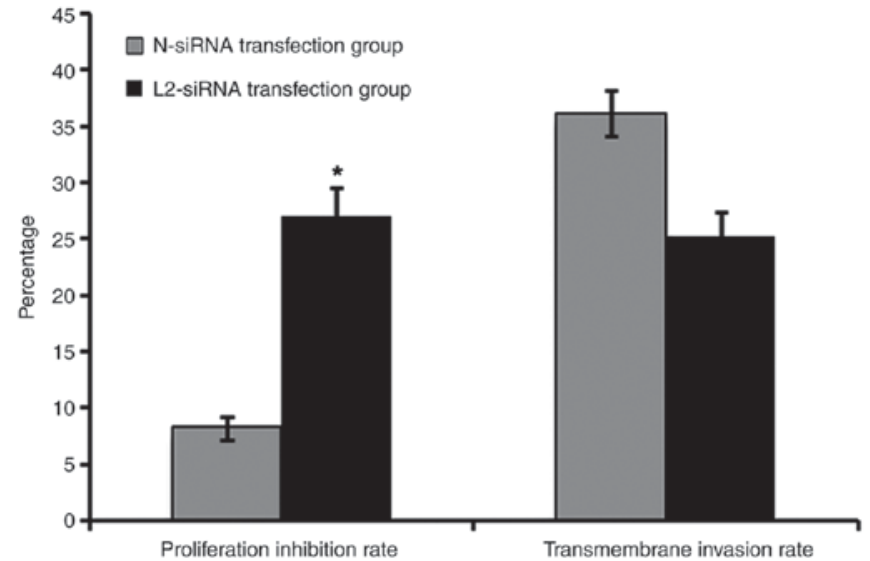

Figure 3. Proliferation inhibition and transmembrane invasion rate of SHI-1 cells were detected following transfection with L2-siRNA and N-siRNA. The inhibition proliferation rate of the L2-siRNA transfection group was $27.043 \pm 2.051 \%$, compared with the negative control group of $8.247 \pm 1.003 \%$ ( $\mathrm{P}<0.05$, compared with the control group). The transmembrane invasion rate of the L2-siRNA transfection group was $25.270 \pm 2.145 \%$ and the invasion rate of the N-siRNA control group was $36.112 \pm 2.103 \%$. siRNA, small interfering RNA; N, negative control.

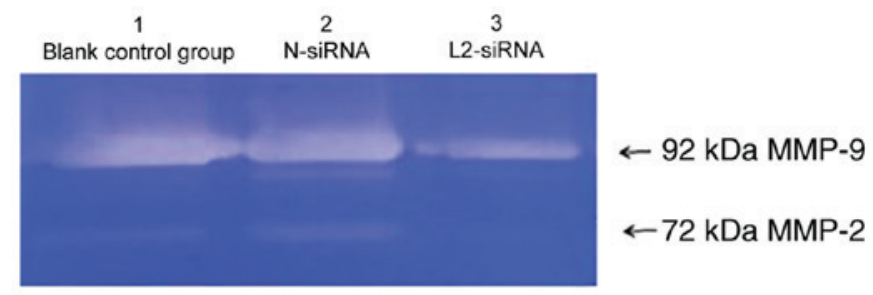

Figure 4. SHI-1 cell culture supernatants were analyzed for the gelatinase levels of MMP-2 and MMP-9 using gelatin zymography following transfection with L2-siRNA (lane 3) and N-siRNA (lane 2) in addition to a blank control group without any siRNA (lane 1). MMP, matrix metallopeptidase; siRNA, small interfering RNA; N, negative control.

unchanged; however, the expression level of p-p38 MAPK and p-JNK was increased in the L2-siRNA transfection group (lane 3) compared with the aforementioned control groups (lanes 1 and 2).

In addition, Photoshop CS3 and SPSS software were used to analyze the results for the expression level of p-MAPK. No difference in the expression levels of ERK, JNK and p38 MAPK between the negative group and the blank control group was identified. However, there were significant differences in the expression level of p-JNK and p-p38 MAPK between the negative group and the L2-siRNA transfection group $(\mathrm{P}<0.05$; Fig. 5B). The increase in the p-JNK and p-p38 MAPK expression level may not be apparent, due to the fact that the transfection efficiency of siRNA in SHI-1 cell suspensions was not high; however, the changes remained statistically significant. Therefore, it was hypothesized that downregulating the expression of LPXN by L2-siRNA activated p-JNK and p-p38 MAPK to inhibit SHI-1 proliferation and invasion.

\section{Discussion}

LPXN is an important cytoskeletal protein that has been identified to be involved in integrin-mediated signal 

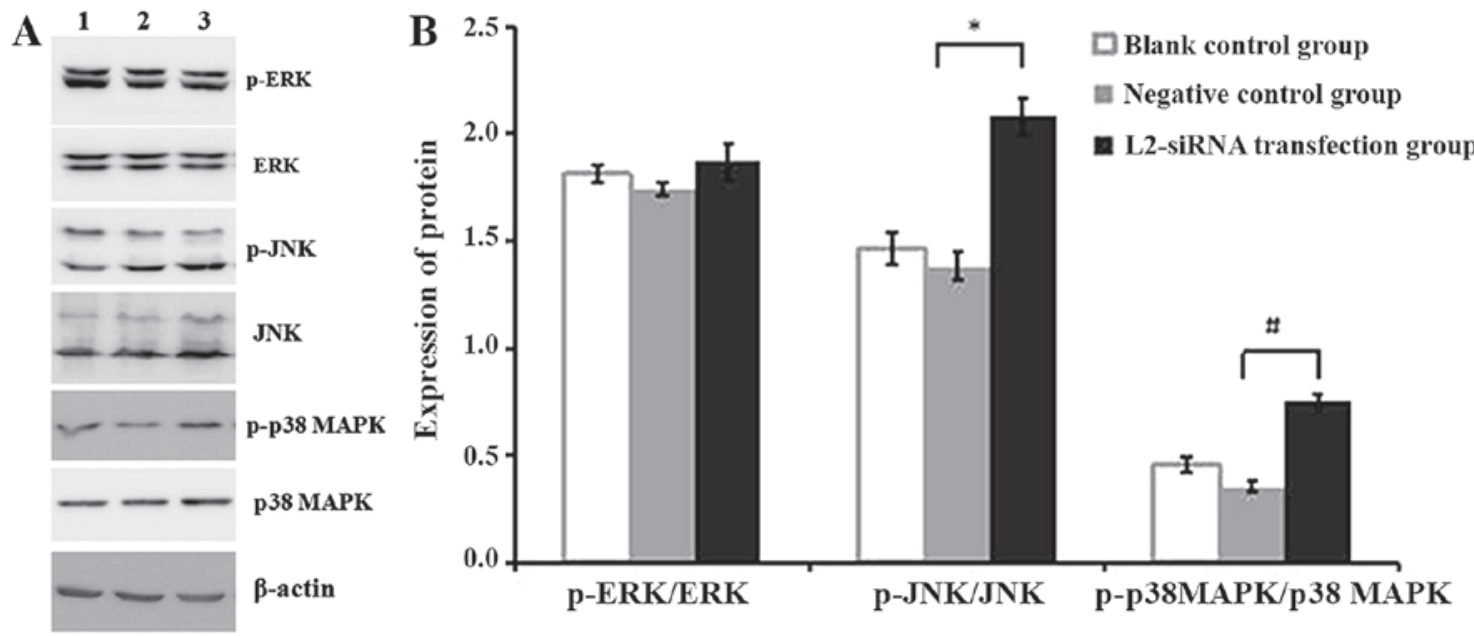

Figure 5. (A) Expression levels of p-ERK, ERK, p-JNK, JNK, p38 MAPK and p-p38 MAPK protein detected by western blot analysis following transfection of SHI-1 cells with L2-siRNA and N-siRNA. Lane 1, blank control group of SHI-1 cells without siRNA; lane 2, negative control group N-siRNA-transfected SHI-1 cells; lane 3, L2-siRNA transfection group of SHI-1 cells. (B) Expression levels of p-ERK, p-JNK and p-p38 MAPK were analyzed by Photoshop CS3, following transfection of SHI-1 cells with L2-siRNA and N-siRNA. The data are expressed as the mean \pm standard deviation of three independent experiments. " $\mathrm{P}<0.05$ vs. the negative control group; ${ }^{\text {}} \mathrm{P}<0.01$ vs. the negative control group. siRNA, small interfering RNA; N, negative control; p, phosphorylated; JNK, c-Jun N-terminal kinase; p38 MAPK, mitogen-activated protein kinase 38; ERK, mitogen-activated protein kinase 1.

transduction $(14,15)$. When integrin binds to its intracellular ligand, the cytoskeletal proteins, involving paxillin, LPXN, talin and vinculin, aggregate to form FAPs and activate FAK. Activated FAK can subsequently trigger downstream signal transduction pathways, such as Ras/MAPK, phosphoinositide 3-kinase (PI3K)/protein kinase B (Akt) and signal transducer and activator of transcription 1 (STAT1), to promote tumor cell proliferation, invasion and to inhibit tumor cell apoptosis (16-19).

Previous research on the function of LPXN in tumors has been primarily focused on prostate cancer. Previous studies, such as Sahu et al (20) and Kaulfuss et al (12), identified that LPXN is highly expressed in PC-3 and DU 145 prostate cancer cells with marked invasiveness, whereas its expression level is relatively decreased in non-invasive prostate cancer LNCaP cells.

It has been demonstrated that downregulating LPXN expression by RNAi in LNCaP cells promotes cancer cell separation and spontaneous apoptosis (12). In addition, it has been identified that interference or knockout of the LPXN gene in the prostate cancer PC-3 and DU 145 cell lines is able to decrease the invasiveness of the aforementioned tumor cell types (21). The specific underlying molecular mechanism in these prostate cancer cells has been reported as follows: LPXN, actin, paxillin and other focal adhesion proteins accumulate in FAPs and interact with FAK, protein tyrosine kinase $2 \beta$, c-Src protein kinases and the protein tyrosine phosphatase non-receptor to activate the downstream signaling pathway involving Ras/MAPK, PI3K/Akt, STAT1, p53 and GTPase, which ultimately increase proliferation, adhesion and invasion in prostate cancer cells $(22,23)$. Similarly, Petti et al (7) revealed that the OSI-930 thiophene inhibitor weakened the activation of Ras/Raf/ERK, PI3K and STAT3/5/6. In addition, it promoted apoptosis in cells and inhibited cellular proliferation, migration and invasion in HMC-1 mast cell leukemia cells by downregulating the phosphorylation of paxillin, LPXN, FAK and Lyn proto-oncogene Src family tyrosine kinase (7).

In the present study, the siRNA sequence that downregulated the high expression of the LPXN gene in SHI-1 cells was selected, and the effects on proliferation and invasion were observed. Using FAM-siRNA and repeatedly transfecting it into SHI-1 cells, it was observed that the optimal transfection conditions included a cell density of $4 \times 10^{5}$ cells $/ \mathrm{ml}$ and a siRNA/Lipofectamine 2000 ratio of $200 \mathrm{pmol} / 1 \mu 1$. The highest transfection efficiency was $74.5 \%$. In addition, the effect of L2-siRNA was examined by western blotting and it was indicated that L2-siRNA decreased the protein expression of LPXN in SHI-1 cells. The present study determined that downregulating LPXN expression by L2-siRNA in SHI-1 cells could inhibit the secretion of MMP-2 and MMP-9, activate JNK and p38 MAPK, and weaken the malignant proliferation and the transmembrane invasive ability of SHI-1 cells in vitro.

Chen et al (24) identified that by increasing the expression of paxillin, proliferation and migration of AGS gastric cancer cells could be upregulated, whereas decreasing the expression of paxillin inhibited the proliferation and migration of SGC7901 gastric cancer cells. In addition, Chew and Lam (25) identified that overexpression of LPXN inhibited the activation of JNK and p38 MAPK in mouse A20 B lymphocytes, thereby attenuating the secretion of interleukin-2 and the function of B cells. The results of the aforementioned studies were consistent with those of the present study, as downregulation of LPXN expression also weakened the proliferation and the transmembrane invasion of leukemic SHI-1 cells, potentially by activating JNK and p38 MAPK. Transfection conditions of FAM-siRNA were optimized using FCM and the effects of L2-siRNA on LPXN expression in SHI-1 cells were screened by western blot analysis. The results of the present study suggested that members of the paxillin family may affect the proliferation and invasion of tumor cells by regulating the activity of MAPK. 


\section{Acknowledgements}

The authors would like to thank the First Affiliated Hospital of Soochow University, who provided the SHI-1 cell lines.

\section{Funding}

The present study was supported by the National Natural Science Foundation of China, Beijing, China (grant no. 81000222) and the Natural Science Foundation for the Youth of Jiangsu, Nanjing, China (grant no. BK20161052).

\section{Availability of data and materials}

The datasets and analyze during the current study are available from the corresponding author on reasonable request.

\section{Authors' contributions}

QS and GHZ designed the experiments and worked on the manuscript. HPD, QZ and GHZ performed the experiments. HPD and QS assisted in the data analysis. All authors discussed the results and commented on the manuscript. All authors read and approved the final manuscript.

\section{Ethics approval and consent to participate}

Not applicable.

\section{Patient consent for publication}

Not applicable.

\section{Competing interests}

The authors declare that they have no competing interests.

\section{References}

1. Lipsky BP, Beals CR and Staunton DE: Leupaxin is a novel LIM domain protein that forms a complex with PYK2. J Biol Chem 273: 11709-22713, 1998

2. Chen PW and Kroog GS: Leupaxin is similar to paxillin in focal adhesion targeting and tyrosine phosphorylation but has distinct roles in cell adhesion and spreading. Cell Adh Migr 4: 527-540, 2010.

3. Deakin NO, Pignatelli J and Turner CE: Diverse roles for the paxillin family of proteins in cancer. Genes Cancer 3: 362-370, 2015.

4. Kaulfuss S, von Hardenberg S, Schweyer S, Herr AM, Laccone F, Wolf S and Burfeind P: Leupaxin acts as a mediator in prostate carcinoma progression through deregulation of p120catenin expression. Oncogene 28: 3971-3982, 2009.

5. Watanabe N, Amano N, Ishizuka H and Mashima K: Leupaxin binds to PEST domain tyrosine phosphatase PEP. Mol Cell Biochem 269: 13-17, 2005.

6. Sundberg-Smith LJ, DiMichele LA, Sayers RL, Mack CP and Taylor JM: The LIM protein leupaxin is enriched in smooth muscle and functions as an serum response factor cofactor to induce smooth muscle cell gene transcription. Circ Res 102: 1502-1511, 2008.
7. Petti F, Thelemann A, Kahler J, McCormack S, Castaldo L, Hunt T, Nuwaysir L Zeiske L, Haack H, Sullivan L, et al: Temporal quantitation of mutant kit tyrosine kinase signaling attenuated by a novel thiophene kinase inhibitor OSI-930. Mol Cancer Ther 4: 1186-1197, 2005.

8. Tanaka T, Moriwaki K, Murata S and Miyasaka M: LIM domain-containing adaptor, leupaxin, localizes in focal adhesion and suppresses the integrin-induced tyrosine phosphorylation of paxillin. Cancer Sci 101: 363- 368, 2010.

9. Dai HP, Xue YQ, Zhou JW, Li AP, Wu YF, Pan JL, Wang Y and Zhang J: LPXN, a member of the paxillin superfamily, is fused to RUNX1 in an acute myeloid leukemia patient with a $\mathrm{t}(11 ; 21)(\mathrm{q} 12 ; \mathrm{q} 22)$ translocation. Genes Chromosomes Cancer 48: 1027-1036, 2009.

10. Abe A, Yamamoto Y, Iba S, Kanie T, Okamoto A, Tokuda M, Inaguma Y, Yanada M, Morishima S, Mizuta S, et al:ETV6-LPXN fusion transcript generated by $\mathrm{t}(11 ; 12)(\mathrm{q} 12.1 ; \mathrm{p} 13)$ in a patient with relapsing acute myeloid leukemia with NUP98-HOXA9. Genes Chromosomes Cancer 55: 242-250, 2016.

11. Wang CL, Chen ZX, Li ZJ and Cen J: The essential roles of matrix metalloproteinase-2, membrane type 1 metalloproteinase and tissue inhibitor of metalloproteinase-2 in the invasive capacity of acute monocytic leukemia SHI-1 cells. Leuk Res 34: 1083-1090, 2010.

12. Kaulfuss S, Grzmil M, Hemmerlein B, Thelen P, Schweyer S, Neesen J, Bubendorf L, Glass AG, Jarry H, Auber B, et al: Leupaxin, a novel coactivator of the androgen receptor, is expressed in prostate cancer and plays a role in adhesion and invasion of prostate carcinoma cells. Mol Endocrinol 22: 1606-1621, 2008.

13. Zhu GH, Dai HP, Shen Q, Ji O, Zhang Q and Zhai YL: Curcumin induces apoptosis and suppresses invasion through MAPK and MMP signaling in human monocytic leukemia SHI-1 cells. Pharm Biol 54: 1303-1311, 2016.

14. Schaller MD: Paxillin: A focal adhesion-associated adapter protein. Oncogene 20: 6459-6472, 2001.

15. van Nimwegen MJ and van de Water B: Focal adhesion kinase: A potential target in cancer therapy. Biochem Pharmacol 73: 597-609, 2007.

16. Calderwood DA, Shattil SJ and Ginsberg MH: Integrins and actin filaments: Reciprocal regulation of cell adhesion and signaling. J Biol Chem 275: 22607-22610, 2000.

17. Omari S: Focal adhesion kinase: A key mediator of cancer pathogenesis. HealthMED 5: 807-818, 2011

18. Sahu SN, Nunez S, Bai G and Gupta A: Interaction of Pyk2 and PTP-PEST with leupaxin in prostate cancer cells. Am J Physiol Cell Physiol 292: C2288-C2296, 2007.

19. Shi J, Wu WJ, Hu G, Yu X, Yu GS, Lu H, Yang ML, Liu B and Wu ZX: Regulation of $\beta$-catenin transcription activity by leupaxin in hepatocellular carcinoma. Tumour Biol 37: 2313-2320, 2016.

20. Sahu SN, Khadeer MA, Robertson BW, Núñez SM, Bai G,and Gupta A: Association of leupaxin with Src in osteoclasts. Am J Physiol Cell Physiol 292: C581-C590, 2007.

21. Kaulfuss S, Herr AM, Büchner A, Hemmerlein B, Günthert AR and Burfeind P: Leupaxin is expressed in mammary carcinoma and acts as a transcriptional activator of the estrogen receptor $\alpha$. Int J Oncol 47: 106-114, 2015.

22. Vanarotti MS, Finkelstein DB, Guibao CD, Nourse A, Miller DJ and Zheng JJ: Structural basis for the interaction between Pyk2-FAT domain and leupaxin LD repeats. Biochemistry 55: 1332-1345, 2016

23. Dierks S, von Hardenberg S, Schmidt T, Bremmer F, Burfeind P and Kaulfuss S: Leupaxin stimulates adhesion and migration of prostate cancer cells through modulation of the phosphorylation status of the actin-binding protein caldesmon. Oncotarget 6: 13591-13606, 2015.

24. Chen DL, Wang ZQ, Ren C, Zeng ZL, Wang DS, Luo HY, Wang F, Qiu MZ, Bai L, Zhang DS, et al: Abnormal expression of paxillin correlates with tumor progression and poor survival in patients with gastric cancer. J Transl Med 11: 277, 2013.

25. Chew V and Lam KP: Leupaxin negatively regulates B cell receptor signaling. J Biol Chem 282: 27181-27191, 2007.

(i) This work is licensed under a Creative Common EY Attribution-NonCommercial-NoDerivatives 4.0 International (CC BY-NC-ND 4.0) License. 